\title{
Smart Agriculture Monitoring System Using IOT
}

\author{
Dr. Sanjay N. Patil ${ }^{1}$, Madhuri B. Jadhav ${ }^{2}$ \\ Associate Professor, Electronics Engg. Department, PVPIT Budhgaon, Sangli, Maharashtra ${ }^{1}$ \\ PG Student, Electronics Engg. Department, PVPIT Budhgaon, Sangli, Maharashtra ${ }^{2}$
}

\begin{abstract}
Agriculture is basic source of livelihood People in India. It plays major role in economy of country. But now days due to migration of people from rural to urban there is hindrance in agriculture. Monitoring the environmental factor is not the complete solution to increase the yield of crops. There are no of factors that decrease the productivity to a great extent. Hence Automation must be implemented in agriculture to overcome these problems. An automatic irrigation system thereby saving time, money and power of farmer. The Traditional Farm land irrigation techniques require manual intervention. With the automated technology of irrigation the human intervention can be minimized. Continuous sensing an monitoring of crops by convergence of sensors with Internet of things (IOT) and making farmers to aware about crops growth, harvest time periodically and in turn making high productivity of crops and also ensuring correct delivery of products to end, consumers at right place and right time. So to overcome this problem we go for smart agriculture technique using IOT. This Project includes sensors such as temperature, humidity, soil moisture and rain detector for collection the field data and processed. These sensors are combined with well established web technology in the form of wireless sensor network to remotely control and monitor data from the sensors.
\end{abstract}

Keywords: Arduino Uno, ESP8266 (Wi-Fi module), Automation of Irrigation System, Sensors.

\section{INTRODUCTION}

Agriculture is major source of income for the largest population in India and is major contributor to Indian economy. In past decade it is observed that there are not much crop development in agriculture sector. Food prices are continuously increasing because crop rate declined. There are number of factor which is responsible for this it may be due to water waste, low soil fertility, Fertilizer abuse, climate change or diseases etc. It is very essential to make effective intervention in agriculture and the solution is IOT in integration with wireless sensor network. Internet of things (IOT) is a method of connecting everything to the internet- it is connecting object or things(such as car, home, electronic devices, etc. ...) which are previously not connected with each other main purpose of IOT is ensuring delivery of right information to right people at right time. In agriculture irrigation is the important factor as the monsoon rain falls are unpredictable and uncertain.

\section{Need of automatic irrigation:}

$>\quad$ Simple an easy to install and configure.

$>\quad$ Saving energy and resources, so that it can be utilized in proper way and amount.

$>\quad$ Farmers would be able to smear to right amount of water at the right time by automatic irrigation.

$>\quad$ Avoiding irrigation at the wrong time of day, reduce runoff from overwatering saturated soils which will improve crop performance.

$>\quad$ Automated irrigation system uses vales to turn motor ON and OFF. Motors can be automated easily by using controllers and no need of labour to turn motor ON an OFF.

$>\quad$ It is precise method for irrigation and a valuable tool for accurate soil moisture control in highly specialized greenhouse vegetable production.

$>\quad$ It is time saving, the human error elimination in adjusting available soil moisture levels.

This project uses IOT technology in agriculture, gathering crops growth environmental parameters in a fixed place to help farmers find problems in time. Agriculture experts give guidelines with specific information to increase the farmer's income and help them in the prevention and control of crop diseases and pests. Through the custom development of mobile phone apps, it has been implemented with agriculture technology promotion and expert online FAQ. The system development composes three parts: The server, Android client and PC client to achieve scalability, high reliability, security, compatibility of technical requirement. 
Vol. 8, Issue 4, April 2019

\section{LITERATURE SURVEY}

Experts have analysed collected data for finding correlation between environment work and yield for standard work. They are concentrated on crop monitoring, information of temperature and rainfall is collected as initial spatial data and analysed to reduce the crop losses and to improve the crop production.

An IOT Based Crop-field monitoring an irrigation automation system explains to monitor a crop field. A system is developed by using sensors and according to the decision from a server based on sensed data, the irrigation system automated. By using wireless transmission the sensed data forwarded towards to web server database. If the irrigation is automated then that means if the moisture and temperature fields fall below the potential range. The user can monitor and control the system remotely with the help of application which provides a web interface to user. [1]

Prof. K.A.Patil and Prof. N.R.Kale propose a wise agricultural model in irrigation with ICT (Information Communication Technology).The complete real-time and historical environment is expected to help to achieve efficient management and utilization of resources. [2]

IOT Based Smart Agriculture Monitoring System develops various features like GPS based remote controlled monitoring, moisture and temperature sensing, intruders scaring, security, leaf wetness and proper irrigation facilities.[3]

Mahammad shareef Mekala, Dr.P.Viswanathan demonstrated some typical application of Agriculture IOT Sensor Monitoring Network Technologies using Cloud computing as the backbone. [4]

Prathibha S.R., Anupama Hongal Jyothi M.P. Created monitoring temperature and Humidity in agriculture field through sensor using CC3200 Single chip. Camera is interfaced with CC3200 to capture images and send that pictures through MMS to farmers mobile using Wi-Fi [5]

\section{PROPOSED WORK}

Block diagram of proposed work:

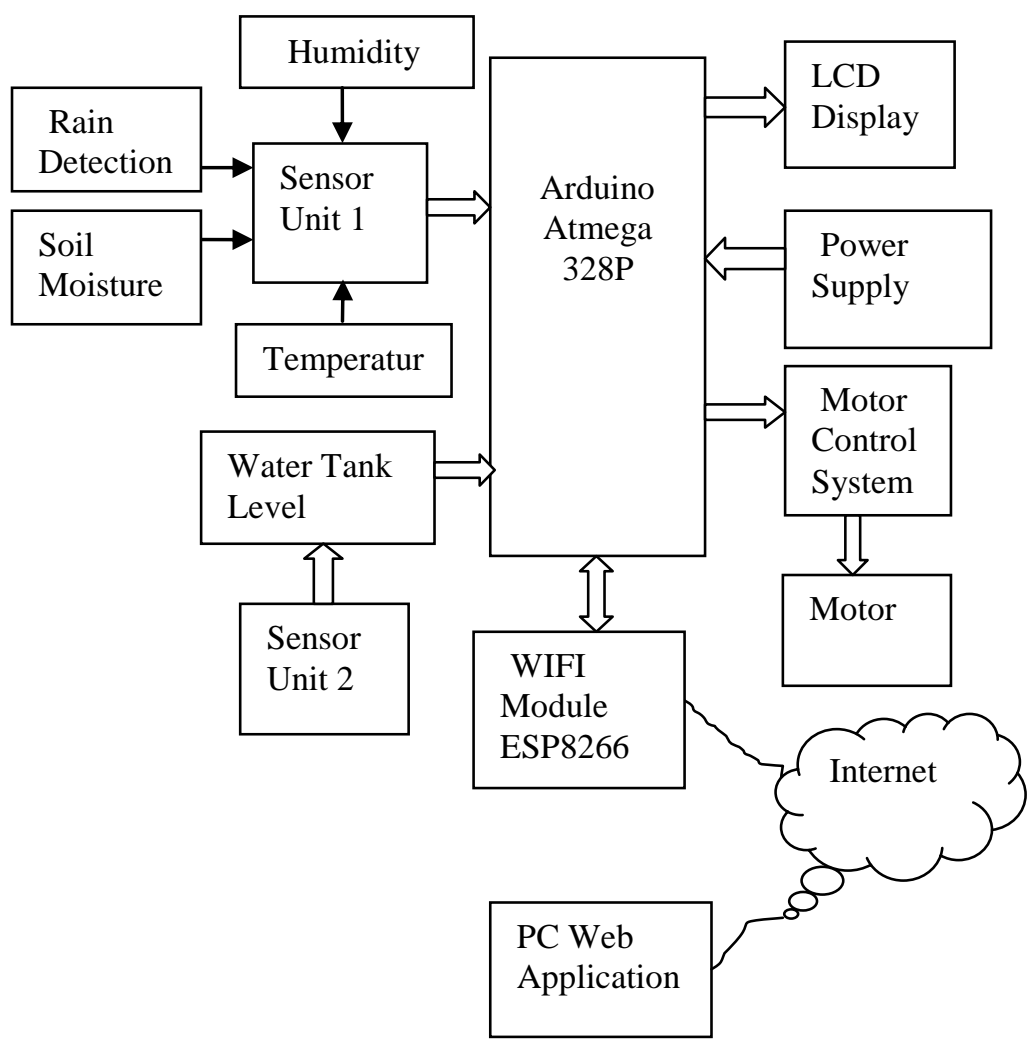

Fig1. Block Diagram Proposed Work 
1) Sensor data acquisition :-

The sensor is interface with Arduino Uno such as DHT11 Temperature, Humidity, Soil moisture and Rain detection sensor is used.

2) Wireless data transmission :ESP8266).

The data acquired from sensors are transmitted to the web server using wireless transmission (WIFI module

3) Data processing and Decision making :-

The data processing is the task of checking various sensors data received from the field with the already fixed threshold values.

The motor will be switched ON automatically if the soil moisture value falls below the threshold and vice- versa. The farmer can even switch ON the Motor from mobile using mobile application.

4) Automation and irrigation system :-

The irrigation system automated once the control received from the web application or mobile application. The relays are used to pass control form web application to the electrical switches using Arduino microcontroller. The circuits with low power signal can be controlled using relay.

\section{5) Web application :-}

The web application will be designed to monitor the field and crops from anywhere using internet connection. To control the Arduino processing IDE is used, the webpage can be communicated using the processing IDE.

\section{6) Mobile Application :-}

The mobile application will be developed in android. The mobile application helps to monitor an controlled filed from anywhere.

\section{IV.HARDWARE USED}

\section{Soil Moisture Sensor:-}

Soil moisture sensor is a sensor which senses the moisture content of the soil. The sensor has both analog and digital output. The digital output is fixed and the analog output threshold can be varied. It works on the principal of open and short circuits. The output is high or low indicated by the LED. When the soil is dry the current will not pass through it and so it will act as open circuit. Hence the output is said to be maximum. When the soil is wet, the current will pass from one terminal to the other and the circuit is said to be short and the output will be zero.

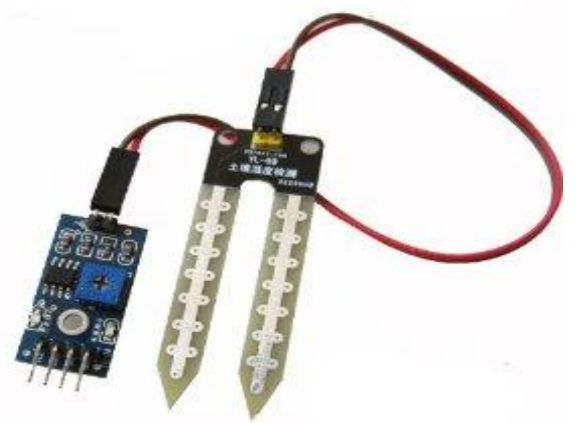

Fig2. Soil moisture sensor

\section{Temperature Sensor:-}

The LM35 sensor is highly used because its output voltage is linear with the Celsius scaling of temperature. It has a wide operating rang. The maximum output is $5 \mathrm{~V}$. The output will increase $10 \mathrm{mV}$ for every one degree rise in temperature. The range is from -55 degrees to +150 degrees. There are three terminals as VCC, Ground and the analog sensor. It consumes minimum amount of electricity. Thus, it is energy efficient. It is every efficient in horticulture. It is user friendly to use. 


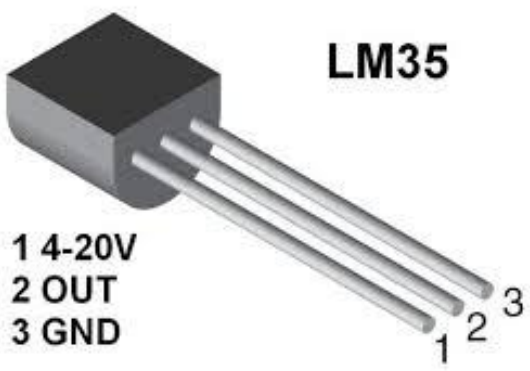

Fig3. Temperature Sensor

\section{Humidity Sensor:-}

Its small size, low power consummation an up-to-20 meter signal transmission making it the best choice for various applications, This DHT11 Humidity sensor features humidity sensor complex with calibrated digital signal output. By using the exclusive digital-signal-acquisition technique and temperature \& humidity sensing technology, it ensures high reliability and excellent long term stability. This sensor includes a resistive -type humidity measurement component.

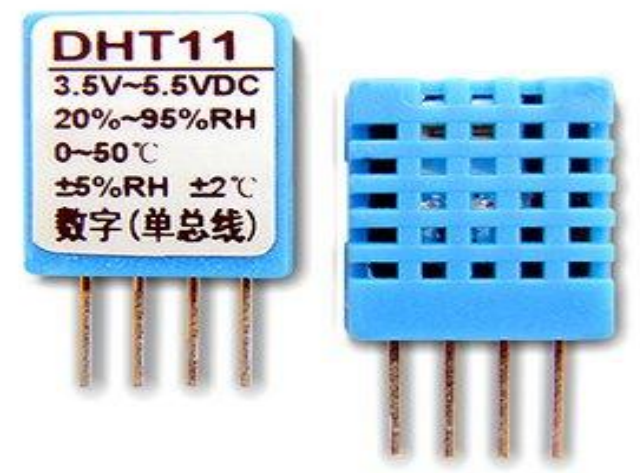

Fig4. Humidity Sensor

\section{Rain Detection Sensor:-}

The rain senor module is an easy tool for rain detection. It can be used as switch when raindrop falls through the raining board and also for measuring rainfall intensity. The module features, a rain board and the control board that is separate for more convenience, power indicator LED and an adjustable sensitivity through a potentiometer. The analog output used in detection of drops in amount of rainfall. Connected to $5 \mathrm{~V}$ power supply, the LED will turn on when induction board has no rain drop, and DO output is high. When dropping a little amount water ,DO output is low, the switch indicator will turn on, Brush off the water droplets, and when restored to the initial state, outputs high level.

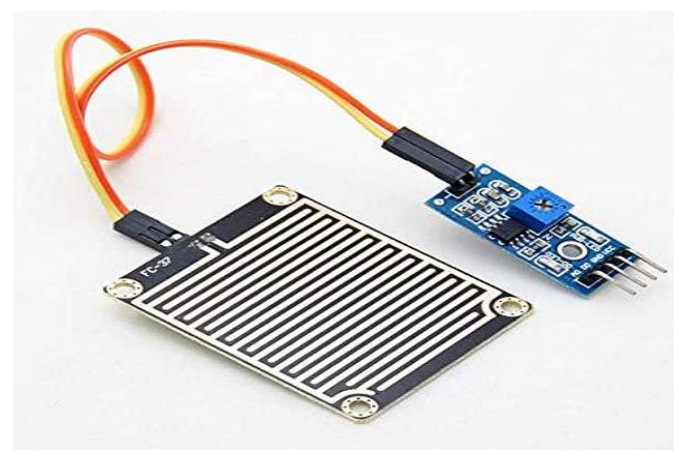

Fig5. Rain Detection Sensor 


\section{Communication Wi-Fi Module:-}

ESP8266 is an impressive, low cost Wi-Fi module suitable for adding Wi-Fi functionality via UART serial communication. Features includes $802.11 \mathrm{~b} / \mathrm{g} / \mathrm{n}$ protocol, Wi-Fi Direct(P2P) soft-AP, Integrated TCP/IP protocol stack.

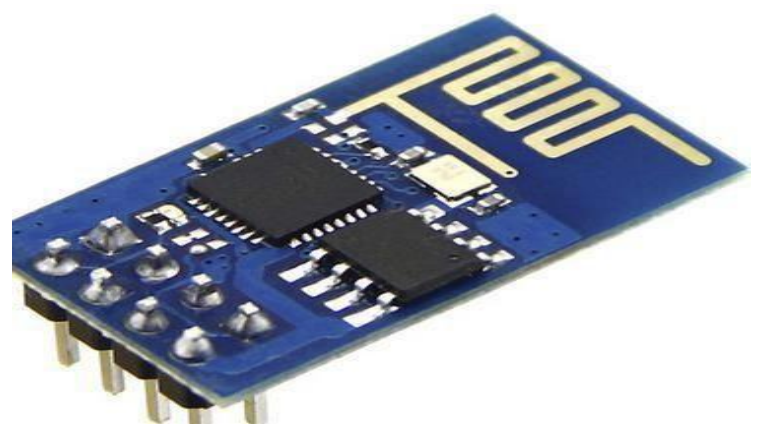

Fig6. Wi-Fi Module

\section{CONCLUSION}

This paper describes automated irrigation system using IOT. Internet on things and cloud computing collectively makes a system that control agriculture sector effectively. This system will sense all the environmental parameters and send the data to the user via cloud. User will take controlling action according to that this will be done by using actuator. This asset allows the farmer to improve the cultivation in a way the plant need. It leads to higher crop yield, prolonged production period, better quality and less use of protective chemicals.

\section{REFERENCES}

[1]. Rajalakshmi.P, Mrs.S.Devi Mahalakshmi "IOT Based Crop-Field Monitoring And Irrigation Automation" 10th International conference on Intelligent systems and control (ISCO), 7-8 Jan 2016 published in IEEE Xplore Nov 2016.

[2]. Prof. K. A. Patil And Prof N. R. Kale proposes "A Model For Smart Agriculture Using IOT” 2016 International Conference on Global Trends in signal Processing, Information Computing And Communication.

[3]. Dr.N.Suma, Sandra Rhea Samson, S. Saranya, G. Shanmugapriya, R. Subhashri 'IOT Based Smart Agriculture Monitoring System' 2017 International Journal on Recent and Innovation Trends in Computing and Communication.

[4]. Mahammad shareef Mekala, Dr.P.Viswanathan 'A Survey: Smart agriculture IoT with cloud Computing' 978-1-5386-1716-8/17/\$31.00 @2017 IEEE

[5]. Prathibha S R1, Anupama Hongal 2, Jyothi M P3' IOT BASED MONITORING SYSTEM IN SMART AGRICULTURE' 2017 International Conference on Recent Advances in Electronics and Communication Technology

[6]. Ibrahim Mat, Mohamed Rawidean Mohd Kassim, Ahmad Nizar Harun, Ismail Mat Yusoff "IOT in Precision Agriculture Applications Using Wireless Moisture Sensor Network” 2016 IEEE Conference on Open Systems (ICOS), October 10-12- 2016, Langkaw, Malaysia.

[7]. Zhaochan Li, JinlongWang, Russell Higgs, LiZhou WenbinYuan4 "Design of an Intelligent Management System for Agricultural Green houses based on the Internet of Things" IEEE International Conference on Embedded and Ubiquitous Computing (EUC) 2017. 